Article

\title{
Power Allocation of Non-Orthogonal Multiple Access Based on Dynamic User Priority for Indoor QoS-Guaranteed Visible Light Communication Networks
}

\author{
Siyu Tao * (1), Hongyi Yu, Qing Li, Xiangwei Bai and Yanqun Tang \\ National Digital Switching System Engineering and Technological Research Center (NDSC), Zhengzhou 450001, \\ China; maxyucn@sohu.com (H.Y.); liqing0206@163.com (Q.L.); bai940102@163.com (X.B.); \\ tangyanqun@126.com (Y.T.) \\ * Correspondence: peachforworking@foxmail.com; Tel.: +86-0371-81622188
}

Received: 25 June 2018; Accepted: 18 July 2018; Published: 25 July 2018

\begin{abstract}
Non-orthogonal multiple access (NOMA) simultaneously provides multi-user access over the same frequency band or time period, which can significantly improve system throughput in visible light communication (VLC) networks. However, the different interference components of NOMA and the diversified user requirements in VLC are difficult to coordinate. To guarantee both throughput enhancement and quality of service (QoS) satisfaction, this paper presents a power allocation scheme based on dynamic user priority in indoor NOMA-VLC networks. We introduce fuzzy logic (FL) to flexibly analyse user priority and assign signal power using multi-dimensional user features. The experimental results show that our method achieves the best performance in terms of user fairness and satisfaction. In indoor VLC, which has small cells with a high user density, our method outperforms contrastive schemes in terms of the average user data rate (AUDR). Therefore, in comparisons between static and dynamic user priority, our study indicates the fairness advantages of FL for the dynamic evaluation of user priority. In comparisons between NOMA and orthogonal multiple access, our study reflects the AUDR advantages of non-orthogonal methods. Furthermore, in comparisons of user satisfaction, our method outperforms existing methods, indicating the achievement of the QoS guarantee.
\end{abstract}

Keywords: optical wireless communication; visible light communication network; non-orthogonal multiple access; power allocation; quality of service guarantee; dynamic user priority; fuzzy logic

\section{Introduction}

The international telecommunications union-radio communications sector (ITU-R) has published the recommendations and key capabilities of next-generation wireless communication, in which the three-type high densities of users, the number of access points (APs) and the area traffic requirement are important performance indicators in the indoor ultra-dense network (UDN) scenario [1]. For illuminations in architecture, optical sources are ubiquitous and the inter-site distance (ISD) of optical APs is much smaller than in traditional cellular or wireless local area networks. Furthermore, the increase in optical AP density with a smaller area of coverage is an efficient way to improve the system traffic capacity, especially in hotspot scenarios [2]. Therefore, to deal with three-type densities, visible light communication (VLC) is a complementary networking scheme of optical wireless communication (OWC) to split cells and improve the spectrum reuse for dense indoor scenes [3].

For high user density, the corresponding user density in typical indoor scenarios, such as at home and in the office, is 0.25 persons per $\mathrm{m}^{2}$ under the cover of each grid [1]. For high AP density, the ISD of optical devices in the office or at home may be less than 10 metres. Compared with mm-wave 
communication, the higher frequencies of OWC may reduce the cell sizes, leading to the concept of LiFi attocells [3], which have a smaller area of coverage than the coverage of femtocells or WiFi. For high traffic requirements, indoor users within different VLC channel conditions may share different communication services with others at the same moment. This leads to a very high traffic density requirement which can reach almost $10 \mathrm{Mbps} / \mathrm{m}^{2}$ [1]. For VLC channels, the system bandwidth of physical optical devices in common markets is limited by the constraint of illuminations, and both illumination and communication functions in indoor VLC networks lead to non-negative signals and strong channel correlations. Therefore, the different quality-of-service (QoS) requirements of VLC users and the efficient spectrum utilization of limited bandwidth should be taken into account in VLC-UDN resource allocation.

Compared with orthogonal multiple access (OMA), non-orthogonal multiple access (NOMA) [4-6] exploits the entire bandwidth for the time-frequency utilization of all degrees of freedom, in order to increase spectral efficiency. In NOMA, the gain ratio power allocation (GRPA) [7] allocates the sorted transmitted power for users with inverse sorted channel gains, which guarantees both fairness and throughput enhancements. In research by Yin et al. [4], analytical solutions for the NOMA-VLC user outage probability in a single cell for guaranteed QoS were derived. In other research by Ding et al. [5], the user was allocated with a higher power allocation coefficient, since the QoS requirements of a user are given by higher priority. With the residual interference from the successive interference cancellation (SIC) of NOMA, user grouping was introduced into the power allocation under the QoS constraints of max-min and max-sum rate criteria for the VLC system throughput enhancement [6]. In a series of studies [7-10], a GRPA strategy based on a power law strategy (PLS) that considers user channel conditions was presented. In PLS, the power allocation of other user gains depends on the gain of the first user, sorted in ascending order, with the assigned power of exponential decrease. The relationship of PLS ensures fairness, whereby a poor channel user will need much higher power due to the large degree of interference. Based on PLS, El-Sayed et al. [11] determined the power coefficients based on a pre-defined QoS per user. High priority users are guaranteed by the signal power allocation based on a pre-defined QoS, while no QoS limitations are defined for lower priority users. In the series of studies $[10,12-15]$, the fractional transmit power allocation (FTPA) was introduced to dynamically adjust the power allocation and reduce the computational complexity compared to full search power allocation. Furthermore, FTPA adopts a fractional power-counting form based on sorted channel gains, which is different from the power counting in PLS. In research by Oviedo and Cao et al. [16,17], when the transmit signal to interference plus noise ratio is not low, the expected NOMA capacity increase following GRPA is greater than the OMA capacity with the same resource allocation conditions, which can be regarded as the guaranteed-QoS data rate. In research by Yapici et al. [18], comprehensive numerical results verified the superiority of NOMA-VLC in achieving a satisfactory QoS target rate by random receiver orientation. Vertical angle information can compensate for loss in user rates by incorporating suitable power allocation coefficients, which are inversely proportional to the channel quality of the respective NOMA user. In summary, the trade-off [19] between reduced user fairness and improved system throughput exists in the user priority and data rate. The aforementioned QoS guarantee methods in VLC networks form at least three types. First, PLS preferentially guarantees high priority users a maximization of throughput which is regarded as the representative method and uses disequilibrium allocation and fixed priority. Second, FTPA weakens the unbalance maximization of throughput which is regarded as the representative method and uses equilibrium allocation and fixed priority. Finally, orthogonal frequency division multiple access (OFDMA) exchanges independent resources for interference mitigation and dynamic priority.

However, in current GRPA studies, several challenges remain in regard to resource allocation with a QoS guarantee for NOMA-VLC networks. First, the user priority of the current GRPA based on the sorted VLC channel gains is static, although power allocation is not static. Second, the evaluation of high user priority depends on QoS requirements alone [5]. Third, VLC channels with NOMA possess a degree of outage vulnerability which is not usually considered in current or recent studies. 
Finally, when the signal demodulation adopts SIC, it is difficult to coordinate different interference components with each user requirement. Hence, user priority should be dynamically evaluated by the integrated state of user communications. In this paper, we consider the fuzzy logic (FL) method with NOMA for two reasons. For service provisions in VLC networks, FL can handle problems involving knowledge representation or definition which are difficult to quantify, such as user priority. On the other hand, Xuan Li et al. [20] indicated that the complexity required to provide full consideration for QoS provision is unaffordable. Yunlu Wang et al. [21] combined a large amount of user state information to determine a suitable load balancing solution with low complexity. In terms of the scheduling in user-specific VLC cells, FL may flexibly handle the complicated user communication information. In regard to the tradeoff between the user data rate and fairness, this paper presents a QoS-guaranteed GRPA based on FL in indoor NOMA-VLC networks. To model the user priority, we introduce three features based on fuzzy logic - the VLC channel, QoS data rate and the NOMA-VLC outage probability-in order to evaluate user priority. Then, the different interference components in each user are comprehensively scheduled by fuzzy logic to ensure balance between the data rate and QoS fairness enhancements. In addition, we consider the outage probability of NOMA-VLC users to induce a realistic numerical simulation by beta distributions. Compared with dynamic OFDMA and static NOMA GRPA methods, such as PLS and FTPA, our design achieves better user fairness and QoS satisfaction than shown in previous studies, while the system throughput of our method is asymptotically optimal under UDN conditions.

\section{Materials and Methods}

\subsection{System Model}

\section{(1) VLC Channel}

In the optical line of sight (LOS) path shown in Figure 1, the VLC channel gain for the indoor $k$-th user is assumed by (1), where $\phi_{k}$ is the angle of irradiation of the light-emitting diode (LED) lights, $\psi_{k}$ is the angle of incidence, $\Phi_{1 / 2}$ is the semi-angle of the LED, $A$ is the received area of a photo diode, $\gamma$ is the optical-electrical conversion efficiency and $T_{f}$ is the gain of the optical filter:

$$
h_{k}=\frac{(m+1) A \gamma}{2 \pi d_{k}^{2}} \cdot \cos ^{m}\left(\phi_{k}\right) \cdot T_{f} \cdot g\left(\psi_{k}\right) \cdot \cos \left(\psi_{k}\right)=\frac{\rho \cdot(m+1) \cdot L^{m+1}}{\left(r_{k}^{2}+L^{2}\right)^{\frac{m+3}{2}}} .
$$
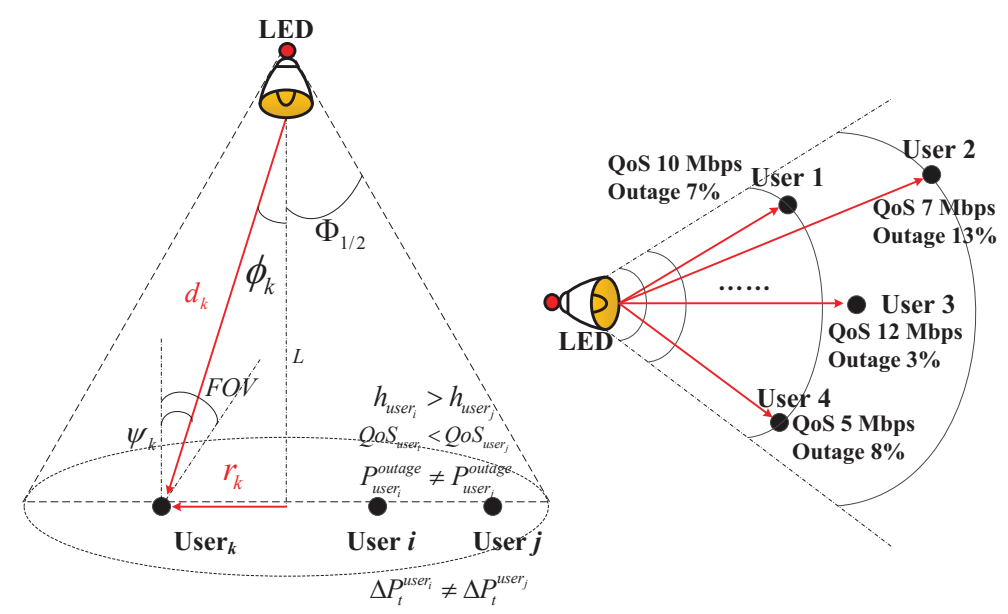

Figure 1. Visible light communication (VLC) user scenario. The labels FOV, LED, and QoS are field of vision, light emitting diode, and quality of service, respectively. 
Let us denote the Lambertian emission order as $m=\frac{\ln 2}{\ln \left(\cos \phi_{1 / 2}\right)}$ and the gain of the optical concentrator as $g\left(\psi_{k}\right)=\frac{n^{2}}{\sin ^{2}\left(\psi_{F O V}\right)}$ with the refractive index of $n$ and the field of view (FOV) $\psi_{F O V}$. In addition, $\rho=\frac{A \cdot \gamma \cdot T_{f} \cdot g\left(\psi_{k}\right)}{2 \pi}$ and $d_{k}^{2}=r_{k}^{2}+L^{2}$. If illumination scenario parameters are given, the VLC channel gain is determined by the distance between the user and the LED.

\section{(2) NOMA GRPA}

In current GRPA studies, both the fairness and efficiency of power resources are considered to follow $h_{1}<h_{2}<\cdots<h_{k}<\cdots<h_{K}$ with $a_{1}>a_{2}>\cdots>a_{k}>\cdots>a_{K}$, where $a_{k}$ is a power allocation factor. If the total signal power for communication denotes $P_{t}$, the sub-channel power $\Delta P_{t}^{u s e r_{k}}$ for each user is allocated by (2) and all of $a_{k}$ are normalized.

$$
\begin{array}{r}
\Delta P_{t}^{\text {user }_{k}}=P_{t} \cdot a_{k}, k<K \\
\operatorname{SINR}_{k}=\frac{\left(h_{k} \cdot a_{k} \cdot P_{t}\right)^{2}}{\sum_{i=k+1}^{K}\left(h_{k} \cdot a_{i} \cdot P_{t}\right)^{2}+N_{0} \cdot B}, k<K \\
C_{k}\left(h_{k}, a_{k}\right)=B \cdot \log _{2}\left(1+\operatorname{SINR}_{k}\right), k<K
\end{array}
$$

Using on-off keying (OOK), the signal to interference plus noise ratio (SINR) and theoretical data rate for the $k$-th user can be determined by (3) and (4). If the positions of users are given, the traditional GRPA introduces the static interference components into each user, which means that each user is prioritised by the pre-defined order. Users with poor channel gains are given higher priority with high signal transmitting power, causing the static resource management of QoS requirements by sorted channel gains.

$$
\begin{aligned}
f_{h_{k}}(h)= & \left|\frac{\partial r^{-1}(h)}{\partial h}\right| \cdot f_{r_{k}}\left(r^{-1}(h)\right) \triangleq F_{1}(h) \cdot F_{2}(h) \\
F_{1}(h)= & \frac{\partial r^{-1}\left(h_{k}^{2}\right)}{\partial h_{k}^{2}}=\frac{1}{\left(\frac{\partial h\left(r_{k}\right)}{\partial r_{k}}\right)} \\
= & \frac{1}{\left(\rho \cdot(m+1) \cdot L^{m+1}\right)^{2} \cdot(-(m+3)) \cdot\left(r_{k}^{2}+L^{2}\right)^{-(m+3)-1} \cdot 2 r_{k}} \\
= & \frac{1}{\left(\rho \cdot(m+1) \cdot L^{m+1}\right)^{2} \cdot(-(m+3)) \cdot\left(\left(\frac{\rho \cdot(m+1) \cdot L^{m+1}}{h_{k}}\right)^{\frac{2}{m+3}}\right)^{-(m+3)-1} \cdot 2 r_{k}} \\
= & -\frac{1}{m+3} \cdot\left(\rho \cdot(m+1) \cdot L^{m+1}\right)^{\frac{2}{m+3}} \cdot\left(h_{k}^{2}\right)^{-\frac{1}{m+3}-1} \cdot \frac{1}{2 r_{k}} \\
F_{h_{k}^{2}}(x)= & \int_{\min \left(h_{k}^{2}\right)}^{\max \left(h_{k}^{2}\right)} f_{h_{k}}(x) d x=\int_{x_{\min }}^{x_{\max }} F_{1}(x) \cdot F_{2}(x) d x \\
= & 1+\frac{L^{2}}{r_{\max }^{2}}-\frac{1}{r_{\max }^{2}} \cdot\left(\rho \cdot(m+1) \cdot L^{m+1}\right)^{\frac{2}{m+3}} \cdot x^{-\frac{1}{m+3}} \\
& x_{\min }=\left[\frac{\rho(m+1) L^{m+1}}{\left(L^{2}\right)^{\frac{m+3}{2}}}\right]^{2} \leq x \leq x_{\max }=\left[\frac{\rho(m+1) L^{m+1}}{\left(r_{\max ^{2}}+L^{2}\right)^{\frac{m+3}{2}}}\right]^{2}
\end{aligned}
$$

\section{(3) NOMA-VLC User Outage Probability}

In NOMA-VLC networks, the user outage probability should be considered because of the vulnerability of optical channels [22,23]. According to the conclusion of (1), we can regard it as $h_{k} \propto \frac{1}{r_{k}^{2}}$ 
and denote $h_{k}$ as the function of $h_{k}\left(r_{k}\right)$. Owing to [6], the probability density function (PDF) $f_{h_{k}}(h)$ of the unordered channel gain can be obtained by the relationship of inverse function $r_{k}{ }^{-1}\left(h_{k}\right)$ in (5).

In (5), we denote $\left|\frac{\partial r^{-1}(h)}{\partial h}\right|$ as $F_{1}(h)$ and denote $f_{r_{k}}\left(r^{-1}(h)\right)$ as $F_{2}(h)$. As in (6), $F_{1}(h)$ can be calculated and $F_{2}(h)=f_{r_{k}}\left(r^{-1}(h)\right)$ can be transformed into $f_{r_{k}}\left(r^{-1}(h)\right)=f_{r_{k}}\left(r_{k}\right)$. Furthermore, $f_{r_{k}}\left(r_{k}\right)$ follows the uniform distribution of users, i.e., $F_{2}(h)=f_{r_{k}}\left(r_{k}\right)=\frac{2 r_{k}}{r_{\max }^{2}}$. Hence, the cumulative distribution function (CDF) of the unordered variable in (7) can be derived from (5) and (6), where $r_{\max }=L \cdot \tan \psi_{F O V}$ and (8) is the field of definitions for CDF.

According to Zhang and Cui et al. [6,24], $C_{k \rightarrow j}\left(h_{k}, a_{j}\right)$ is denoted as the rate of (4) for the $k$-th user to detect the $j$-th user message $(j \leq k)$. When the outage occurs, the $k$-th user is not able to obtain their own message, which yields (9) to indicate the outage event.

$$
\begin{aligned}
C_{k \rightarrow j}\left(h_{k}, a_{j}\right) & =B \cdot \log _{2}\left(1+\frac{\left(h_{k} a_{j} P_{t}\right)^{2}}{\sum_{i=j+1}^{K}\left(h_{k} a_{i} P_{t}\right)^{2}+N_{0} B}\right) \geq C_{j}\left(h_{j}, a_{j}\right), j \leq k \\
P_{k}^{\text {out }} & =1-P\left(C_{k \rightarrow j} \geq C_{j}, \forall j \leq k\right)=1-P\left(h_{k}^{2} \geq \varepsilon_{k}^{*}, \forall j \leq k\right) \\
& =\sum_{i=k}^{K} \frac{K !}{i !(K-i) !} F_{h_{k}^{2}}\left(\varepsilon_{k}^{*}\right)^{i}\left[1-F_{h_{k}^{2}}\left(\varepsilon_{k}^{*}\right)\right]^{K-i} \\
\text { Rate }_{k}\left(h_{k}, a_{k}, P_{k}^{\text {out }}\right) & =\left(1-P_{k}^{\text {out }}\right) \cdot B \cdot \log _{2}\left(1+\operatorname{SINR} R_{k}\right), k<K .
\end{aligned}
$$

Using order statistics [6], the outage probability, $P_{k}^{\text {out }}$, for the $k$-th user can be indicated as (10), where $\varepsilon_{k}^{*}$ is a threshold in the field of definitions, as (8) shows. Finally, the achievable user data rate is presented in (11), and our resource allocation target is to maximize the sum user data rate (i.e., system throughput) and consider the desired user data rate (i.e., QoS requirements) through rational power allocation. We note that the PDF in (5) and CDF in (7) are unordered. However, the threshold, $\varepsilon_{k}^{*}$, is difficult to decide on and (10) is ordered, which may not be directly available for dynamic user priority.

\subsection{Dynamic User Priority}

Figure 1 shows that different users yield different requirements which cannot be decided by channel state information alone. Considering NOMA in VLC networks, the three typical aspects including the special channel state, NOMA interruption probability and different QoS requirements are key features, which jointly influence the quality of services.

This paper introduces the fuzzy logic (FL) system and balances three types of variables, namely, the instantaneous channel gain of a VLC user, the QoS data rate requirement desired by users and the outage of a NOMA-VLC user in a single cell. It is assumed that the FL system is deployed in the center unit of the optical AP. In Table 1, each type of input is represented by three grades-low, medium and high-except for the complex QoS data rate requirement which has more than three grades. The diagram of the fuzzy logic scheme is indicated in Figure 2.

Table 1. The grades of membership fuzzification. The labels NOMA and VLC are non-orthogonal multiple access and visible light communication, respectively.

\begin{tabular}{cc}
\hline Input Variables of Three Features & Grades \\
\hline VLC channel gain & (Low, Medium, High) \\
User desired data rate & (Low, Low-Medium, Medium-High, High) \\
NOMA-VLC outage probability & (Low, Medium, High) \\
\hline
\end{tabular}




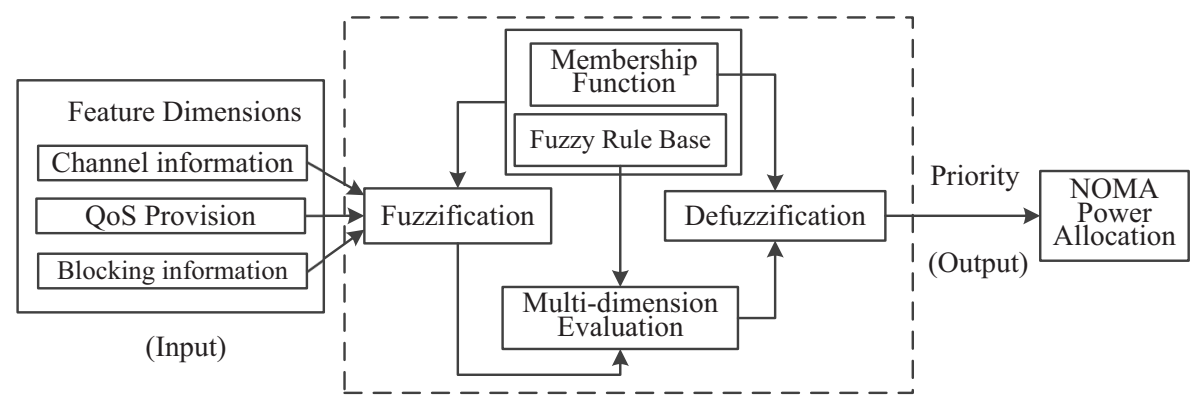

Figure 2. Fuzzy logic scheme. The labels NOMA and QoS are non-orthogonal multiple access and quality of service, respectively.

\section{(1) Fuzzification}

The fuzzification converts input variables of the fuzzy logic (FL) system into linguistic variables with membership functions (MFs). For each variable, the MF is designed to map the value to its corresponding desirableness membership. Considering that a triangular membership function is a special case in trapezoidal membership, we use the trapezoidal membership of (12) in our NOMA-VLC FL system to give a fine-grained and diversified degree of characterization. Note that the grades are denoted as $\mathbf{G}$ and $G \in \mathbf{G}$ in (13). If any two adjacent variables in $\left(a_{0}^{(G)}, b_{0}^{(G)}, c_{0}^{(G)}, d_{0}^{(G)}\right)$ are equal, the output of the MFs in the definition field between two identical-value adjacent variables is equal to one.

$$
\begin{gathered}
M F_{(G)}\left(x ; a_{0}^{(G)}, b_{0}^{(G)}, c_{0}^{(G)}, d_{0}^{(G)}\right)=\left\{\begin{array}{cc}
0, & x \leq a_{0}^{(G)} \\
\frac{x-a_{0}^{(G)}}{b_{0}^{(G)}-a_{0}^{(G)}}, & a_{0}^{(G)} \leq x \leq b_{0}^{(G)} \\
1, & b_{0}^{(G)} \leq x \leq c_{0}^{(G)} \\
\frac{d_{0}^{(G)}-x}{d_{0}^{(G)}-c_{0}^{(G)},} & c_{0}^{(G)} \leq x \leq d_{0}^{(G)} \\
0, & x \geq d_{0}^{(G)}
\end{array}\right. \\
{\left[a_{0}^{(G)}, b_{0}^{(G)}, c_{0}^{(G)}, d_{0}^{(G)}\right]_{G \in \mathbf{G}}=\left[\begin{array}{ccc}
a_{0}^{(G=\text { Low })} & \cdots & d_{0}^{(G=\text { Low })} \\
\vdots & \ddots & \vdots \\
a_{0}^{(G=\text { High })} & \cdots & d_{0}^{(G=\text { High })}
\end{array}\right]_{|\mathbf{G}| \times 4} .}
\end{gathered}
$$

(2) Fuzzy Rule Base

When the priority of a user is indicated by the MF outputs of each feature, the priority should be dynamically decided by three grades (i.e., high, medium and low, as shown in Table 2. Each rule uses the logic operation (i.e., logic operation and) and describes the multi-level membership degree of a user priority. Note that the larger membership degree of priority from the same grade should be selected.

\section{(3) Defuzzification}

Using the gravity centre method, each degree of user priority can be substituted into (14). The use of MFs in (14) can be designed as similar as in (12), transforming the linguistic degree of prioritisation into the score value. When the priority of a user is indicated in different grades of membership degrees, (14) turns the multiple grades into the final score, corresponding to the power allocation factor. 
Table 2. Fuzzy rule base.

\begin{tabular}{ccccc}
\hline No. & VLC Channel Gain & Desired Rate & Outage Probability & User Priority \\
\hline 1 & - & High & Low & High \\
2 & Not Low & Med-High & Low & High \\
3 & - & High & Not Low & Med \\
4 & High & Med-High & Not Low & Med \\
5 & Low & Med-High & Low & Med \\
6 & - & Low-Med & Low & Med \\
7 & Not High & Med-High & High & Low \\
8 & - & Low-Med & High & Low \\
9 & - & Low & - & Low \\
\hline \multicolumn{5}{c}{} \\
\multicolumn{5}{c}{$\lambda_{k}=\frac{\int_{0}^{1} M F(x) x d x}{\int_{0}^{1} f(x) d x}}$. \\
\end{tabular}

\subsection{QoS-Guaranteed NOMA-VLC GRPA}

Due to the reasons outlined in the discussion of NOMA-VLC user outage probability, dynamic user priority and difficult threshold selection affect the outage distribution. According to reference [4], the threshold $\varepsilon_{k}^{*}$ of (10) is given by $\varepsilon_{k}^{*}=\min \left\{\max \left\{\varepsilon_{1}, \cdots, \varepsilon_{k}, \cdots, x_{\min }\right\}, x_{\max }\right\}$, where $x_{\min }$ and $x_{\max }$ are in (8). However, we notice that the determination of $\varepsilon_{k}^{*}$, defined by [4], may not be easy to search. We propose using the beta distribution to estimate the original distribution for convenience in the observation of dynamic user priority. In approximate equivalence, the idea that we transform the determination of $\varepsilon_{k}^{*}$ into the number of users is further deduced:

$$
\sum_{i=k}^{K} \frac{K !}{i !(K-i) !} F_{h_{k}^{2}}(x)^{i}\left[1-F_{h_{k}^{2}}(x)\right]^{K-i}=\frac{K !}{(i-1) !(K-i) !} \int_{0}^{F_{h_{k}^{2}}(x)} t^{i-1}(1-t)^{K-i} d t
$$

It is clear that the left and right formulas in (15) are identical. If (10) is indicated by (15), the PDF of the outage can be expressed as (16):

$$
\begin{aligned}
f_{P_{k}^{\text {out }}}(x) & =\left[P_{k}^{\text {out }}\right]^{\prime}=\frac{\Gamma(K+1)}{\Gamma(i) \cdot \Gamma(K+1-i)} F_{h_{k}^{2}}(x)^{i-1} \cdot\left[1-F_{h_{k}^{2}}(x)\right]^{K-i} \cdot f_{h_{k}^{2}}(x) \\
& =\frac{F_{h_{k}^{2}}(x)^{i-1} \cdot\left[1-F_{h_{k}^{2}}(x)\right]^{K-i} \cdot f_{h_{k}^{2}}(x)}{\operatorname{Be}(i, K+1-i)} \propto f_{h_{k}^{2}}(x) \cdot \operatorname{Beta}\left(F_{h_{k}^{2}}(x) ; i, K+1-i\right) .
\end{aligned}
$$

If $B e(\alpha, \beta) \triangleq \frac{\Gamma(\alpha+\beta)}{\Gamma(\alpha) \cdot \Gamma(\beta)}, \Gamma(n) \triangleq(n-1) !$ and $f_{h_{k}^{2}}(x)=F^{\prime} h_{k}^{2}(x),(17)$ is obtained. When uthe ser positions are fixed, the distributions of $F_{h_{k}^{2}}(x)$ and $f_{h_{k}^{2}}(x)$ are determined, and we can perform an approximate numerical calculation in which the beta distribution can be used as the estimation of the outage probability distribution. For example, the probability, $P_{k}^{\prime \text { out }}$, of a discrete event can be generated by the normalization of $\operatorname{Beta}(i, K+1-i)$ in the numerical simulation. The accessible user data rate based on the dynamic user priority is formulated by (18):

$$
\text { Rate }_{k}\left(h_{k}, a_{k},{P_{k}^{\prime o u t}}^{\text {out }} \lambda_{k}\right)=\left(1-P_{k}^{\prime \text { out }}\right) \cdot B \cdot \log _{2}\left(1+\frac{\left(h_{k} \lambda_{k} P_{t}\right)^{2}}{\sum_{i \in[1, K]}^{\sum_{i}<\lambda_{k}}\left(h_{k} \lambda_{i} P_{t}\right)+N_{0} B}\right), k<K
$$




\section{Results and Discussion}

\subsection{Experimental Conditions and Parameters}

For a room of $6 \mathrm{~m} \times 6 \mathrm{~m} \times 3 \mathrm{~m}$, the parameters of the VLC scenario are indicated in Table 3 . According to indoor UDN modeling, a high user density is 0.25 persons per square meter, so the critical value of the number of ultra-dense users is nine persons in the simulation scenario. For the probability distribution generation of QoS, the gamma distribution is selected to set the QoS expectation $\left(\alpha_{1} \cdot \beta_{1}\right) \in$ $\{1,10,20,30,40,50,60\}$ (Mbps), where $\left(\alpha_{1} \cdot \beta_{1}\right) \in\{1 \times 1,2 \times 5,4 \times 5,5 \times 6,5 \times 8,5 \times 10,6 \times 10\}$. For the estimation of the outage probability, if we denote the number of users as $K$, the normalization of the beta distribution is selected to set $\alpha_{2}=K+1$ and $\beta_{2}=k, k \leq K$.

Table 3. Experimental parameters.

\begin{tabular}{cccc}
\hline Symbol & Value & Symbol & Value \\
\hline Received area & $10^{-4} \mathrm{~m}^{2}$ & Received height & $L=1.8 \mathrm{~m}$ \\
$\psi_{F O V}$ & $60^{\circ}$ & $\Phi_{1 / 2}$ & $60^{\circ}$ \\
$P_{t}$ & $8 \mathrm{~W}$ & $\gamma$ & 0.47 \\
Bandwidth & $25 \mathrm{MHz}$ & $N_{0}$ & $10^{-19} A^{2} / \mathrm{Hz}$ \\
$T_{f}$ & 1 & $n$ & 1.5 \\
QoS & $\sim \Gamma\left(\alpha_{1}, \beta_{1}^{-1}\right)$ & outage & $\sim \operatorname{Beta}\left(\alpha_{2}, \beta_{2}\right)$ \\
\hline
\end{tabular}

In the current research, NOMA PLS and NOMA FTPA are the typical power allocation strategies, and our proposed power allocation method is denoted as NOMA FL. To confirm that NOMA FL guarantees the QoS as much as possible, we regard the upper data rate bound of OMA as the QoS requirements of the NOMA system to reflect the data rate relationship among NOMA FL, PLS and FTPA. For verification that NOMA FL satisfies dynamic user priority, the comparisons include two aspects:

- $\quad$ To determine the performance difference between static and dynamic user priorities, we performed comparisons among NOMA FL, NOMA PLS and NOMA FTPA.

- $\quad$ To demonstrate the performance difference between NOMA and OMA, we measured NOMA FL and OFDMA FL with the same dynamic user priority.

The measurement of advantages of the FL system did not uniquely involve the user data rate $[25,26]$, which may not synthetically reflect the performance of the FL system. For the measurement of adaptability to QoS guarantee in VLC networks, as shown in Table 4, we performed three simulation cases to analyse the performance of NOMA FL, NOMA PLS, NOMA FTPA, and OFDMA FL. The purpose of each case and the evaluation indicators are listed as follows.

- $\quad$ Case 1 (analysis of FL dimensions): Without considering QoS requirements, PLS and FTPA can equivalently be regarded as two-dimension considerations of the channel gain and outage. For two dimensions of FL features, we performed experiments regarding the user data rate and user satisfaction in NOMA FL and OFDMA FL without QoS constraints. In this case, we discovered that PLS and FTPA cannot successfully guarantee the QoS, and that the maximization of throughput is not equivalent to the provision of QoS data rate.

- $\quad$ Case 2 (analysis of the variety of QoS): For the three complete FL features, comparing PLS with FTPA, NOMA FL revealed the difference between dynamic and static user priorities. In regard to the comparison of NOMA FL with the dynamic user priority of OFDMA FL, NOMA FL was able to accommodate QoS requirements to show a difference in system performance. In this case, regarding the tradeoff between user data rate and QoS guarantee, we introduced the commonly used evaluation measure of QoS guarantee to demonstrate the clear advantages of FL. 
- Case 3 (analysis of VLC lighting): In different visible lighting conditions, we showed the relationship between the FOV angle of illumination and the performance of the communication schemes. In this case, combined with VLC UDN, we considered both high user density and area user traffic demands to show the advantage of FL.

- Evaluation indicators: the average user data rate (AUDR), Jain fairness [27], max-min fairness [5,27] and user satisfaction [25] were introduced. Jain fairness was used to evaluate the impartiality of the user data rate and max-min fairness focused on the guaranteed baseline of the QoS data rate. User satisfaction $[21,28]$ is a comprehensive evaluation indicator that consides both data rate and fairness.

Table 4. Comparisons of different cases. The labels FL, FOV, FTPA, NOMA, OFDMA, PLS, and QoS are fuzzy logic, field of vision, fractional transmit power allocation, non-orthogonal multiple access, orthogonal frequency division multiple access, power law strategy, and quality of service, respectively.

\begin{tabular}{cccc}
\hline Description & Case 1 & Case 2 & Case 3 \\
\hline Influence factor & FL dimensions & QoS, & Layout \\
Features & $h_{k}, P_{k}^{\text {out }}$ & $h_{k}$, QoS, $P_{k}^{\text {out }}$ & with light angles \\
\hline NOMA FL & Dynamic $\left(h_{k}, P_{k}^{\text {out }}\right)$ & Dynamic $\left(h_{k}\right.$, QoS, $\left.P_{k}^{\text {out }}\right)$ & $\psi_{F O V}$ \\
NOMA PLS & Static $\left(h_{k}, P_{k}^{\text {out }}\right)$ & Static $\left(h_{k}, P_{k}^{\text {out }}\right)$ & $\psi_{F O V}$ \\
NOMA FTPA & Static $\left(h_{k}, P_{k}^{\text {out }}\right)$ & Static $\left(h_{k}, P_{k}^{\text {out }}\right)$ & $\psi_{F O V}$ \\
OFDMA FL & Dynamic $\left(h_{k}, P_{k}^{\text {out }}\right)$ & Dynamic $\left(h_{k}\right.$, QoS,$\left.P_{k}^{\text {out }}\right)$ & $\psi_{F O V}$ \\
\hline
\end{tabular}

\subsection{Case 1: Experimental Analysis in FL dimensions}

When the MFs were set to (19) and (20) with the vector norm, $\mathbf{h}$, including the channel gains of all users, Figure 3 shows that the local effectiveness of NOMA FL reached the data rate bound of PLS in the case of high user density. Without QoS features, the AUDR depends on channel gains and outages, which are prone to having a single target in throughput maximization.

$$
\begin{gathered}
{\left[a_{0}^{(G)}, b_{0}^{(G)}, c_{0}^{(G)}, d_{0}^{(G)}\right]_{h, G \in[L, M, H]^{\mathrm{T}}}=\left[\begin{array}{cccc}
0 & 0.1 & 0.3 & 0.3 \\
0.1 & 0.3 & 0.6 & 0.8 \\
0.6 & 0.8 & 0.8 & 0.8
\end{array}\right] \times|\mathbf{h}|_{\infty}} \\
{\left[a_{0}^{(G)}, b_{0}^{(G)}, c_{0}^{(G)}, d_{0}^{(G)}\right]_{\text {outage, } G \in[L, M, H]^{\mathrm{T}}}=\left[\begin{array}{cccc}
0 & 0.05 & 0.1 & 0.1 \\
0.1 & 0.15 & 0.2 & 0.25 \\
0.2 & 0.25 & 0.35 & 0.45
\end{array}\right]} \\
{\left[a_{0}^{(G)}, b_{0}^{(G)}, c_{0}^{(G)}, d_{0}^{(G)}\right]_{\mathrm{QoS}, G \in[L, L M, M H, H]^{\mathrm{T}}}=\left[\begin{array}{cccc}
0 & 0 & 0.25 & 0.25 \\
0.05 & 0.25 & 0.5 & 0.5 \\
0.5 & 0.7 & 1.0 & 1.0 \\
1.0 & 1.5 & 2.0 & 2.0
\end{array}\right] \times\left|\alpha_{1} \cdot \beta_{1}\right| .}
\end{gathered}
$$

In Figure 3, PLS and FTPA are treated as two-dimension methods, and NOMA FL and OFDMA FL are related to two-dimension FL features. When the MF of the QoS requirements was set to (21), as shown in Figure 4, the holistic high QoS requirement may lead to unreasonable resource allocation and a decrease in the system throughput. Although the QoS parameters were set, the PLS and FTPA based on static user priority may, theoretically, not consider the QoS. Hence, the differences between $30 \mathrm{Mbps}$ and $60 \mathrm{Mbps}$ QoS expectations, based on PLS and FTPA, are caused by the channel gain distribution of different user positions.

In this experiment, the critical value of high user density in indoor VLC UDN was nine users, which was obtained by 0.25 persons $/ \mathrm{m}^{2}$ and 36 square meters. Furthermore, when $\psi_{F O V}=60^{\circ}$ and $L=1.8 \mathrm{~m}$, as shown in Table 3, an optical AP covered a smaller area than $36 \mathrm{~m}^{2}$, which is 
approximate to $27 \mathrm{~m}^{2}$. In Figure 3, the peak value of high area traffic requirement is shown to reach $10 \mathrm{Mbps} / \mathrm{m}^{2}$, which means that NOMA FL can handle indoor VLC cells. With different dimensions of Figures 3 and 4, we showed that the multi-dimension FL of NOMA outperforms other methods under VLC UDN conditions which are used in our proposed scenario.

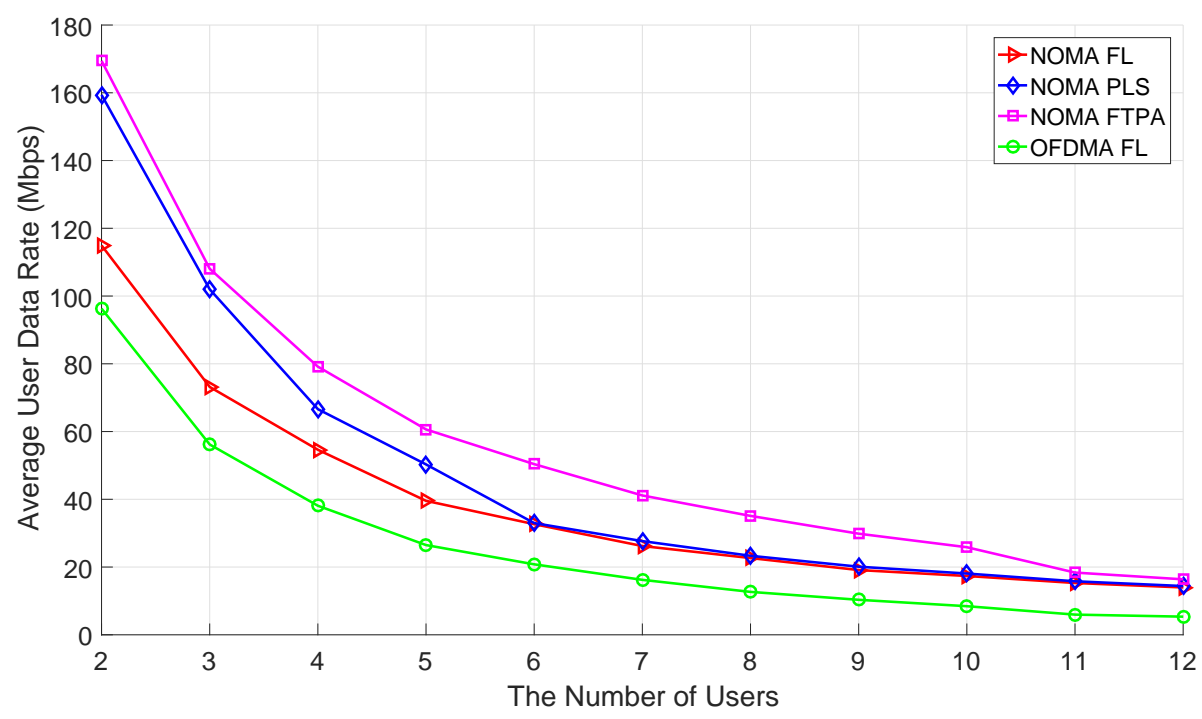

Figure 3. Comparisons of the average user data rate (AUDR) in two dimensions without quality-of-service (QoS) features. The labels FL, FTPA, NOMA, OFDMA, and PLS are fuzzy logic, fractional transmit power allocation, non-orthogonal multiple access, orthogonal frequency division multiple access, and power law strategy, respectively.

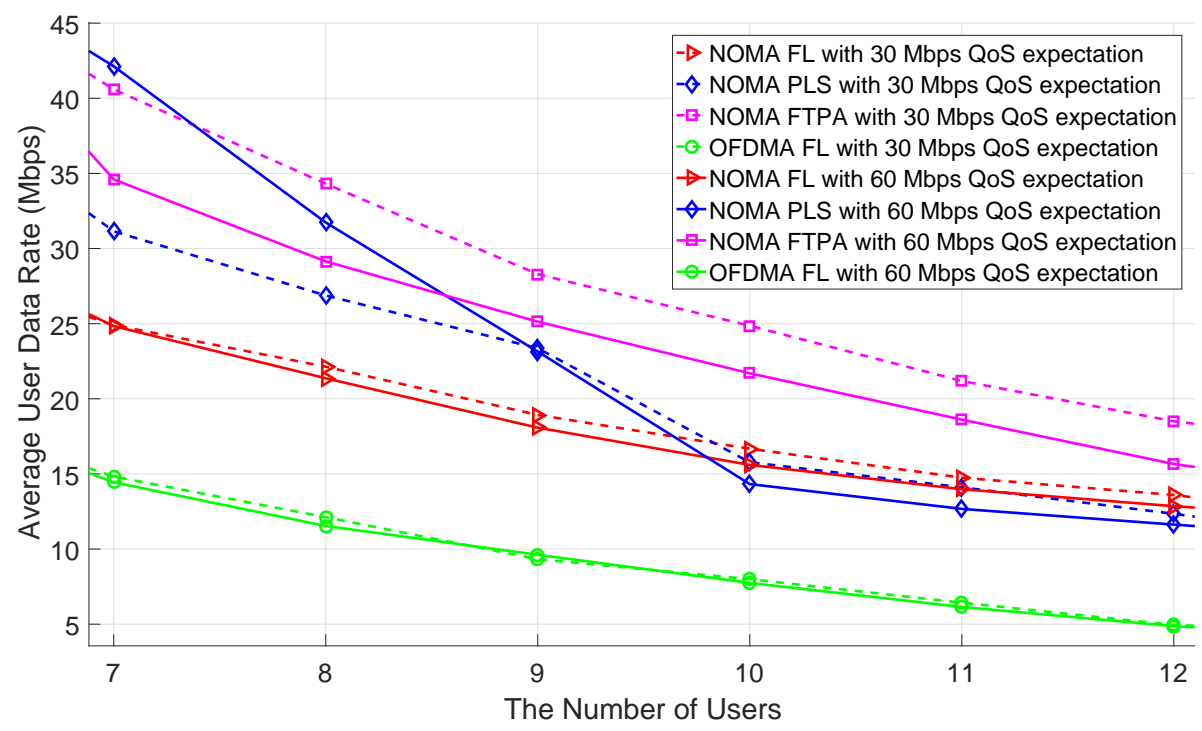

Figure 4. Comparisons of the AUDR in three dimensions with varied users and QoS expectations of $30 \mathrm{Mbps}$ and 60 Mbps.

\subsection{Case 2: Experimental Analysis of QoS Guarantee}

\subsubsection{User Satisfaction}

On the surface of the AUDR, PLS and FTPA appear better than NOMA FL and OFDMA FL. However, the purpose of this paper is QoS-guaranteed, and Figure 3 cannot reveal the defects of PLS and FTPA for two reasons: 
- The tradeoff between the AUDR and QoS guarantee exists in VLC networks. Only AUDR cannot reflect the performance of QoS provision.

- The measurement of AUDR is one-sided, which is not enough to indicate the degree of users QoS satisfaction. To discuss the QoS guarantee, user satisfaction should be considered to comprehensively evaluate the performance of FL in QoS provision.

We obtained Figure 5 to indicate the defects of PLS and FTPA, which are unable to meet QoS demands. References $[21,28]$ show that the ratio of the AUDR to QoS requirement is related to user satisfaction. Hence, the high performance of AUDR is not enough to provide full consideration for demands of all users. Although Figure 3 shows the high AUDR in PLS and FTPA, Figure 5 indicates that PLS and FTPA are not effective for QoS guarantee.

In Figure 6, we use the indicator of user satisfaction for integrated assessment of both the user data rate and the QoS guarantee of fairness. First, Figure 6 indicates the exceptional variety of PLS $\mathrm{CDF}$, caused by the strategy of PLS and leading to the polarization of both the power allocation and the guaranteed QoS data rate. Second, when $K=4$, the user satisfaction of NOMA FL was better than the user satisfaction of the other methods in $K=9$. The situations in $K=4$ were better than those of $K=9$ because $K=4$ is smaller than the critical value of the ultra-dense user number and is not overloaded for resource allocation. Third, the maximum performance gap in $K=4$ was greater than that in $K=9$. The user satisfaction gap between FTPA and NOMA FL decreased when the number of users grew. Under the same QoS expectation, a growing number of users may cause limited resource allocation which may result in the deficiency of the QoS guarantee. Finally, in terms of static and dynamic user priority, NOMA FL outperformed PLS and FTPA, and OFDMA FL also outperformed NOMA PLS. This means that our FL system is effective for modeling user priority and achieving the QoS guarantee. In regard to NOMA and OMA, NOMA FL was better than OFDMA FL, which means that NOMA improves the user data rate and QoS guarantee.

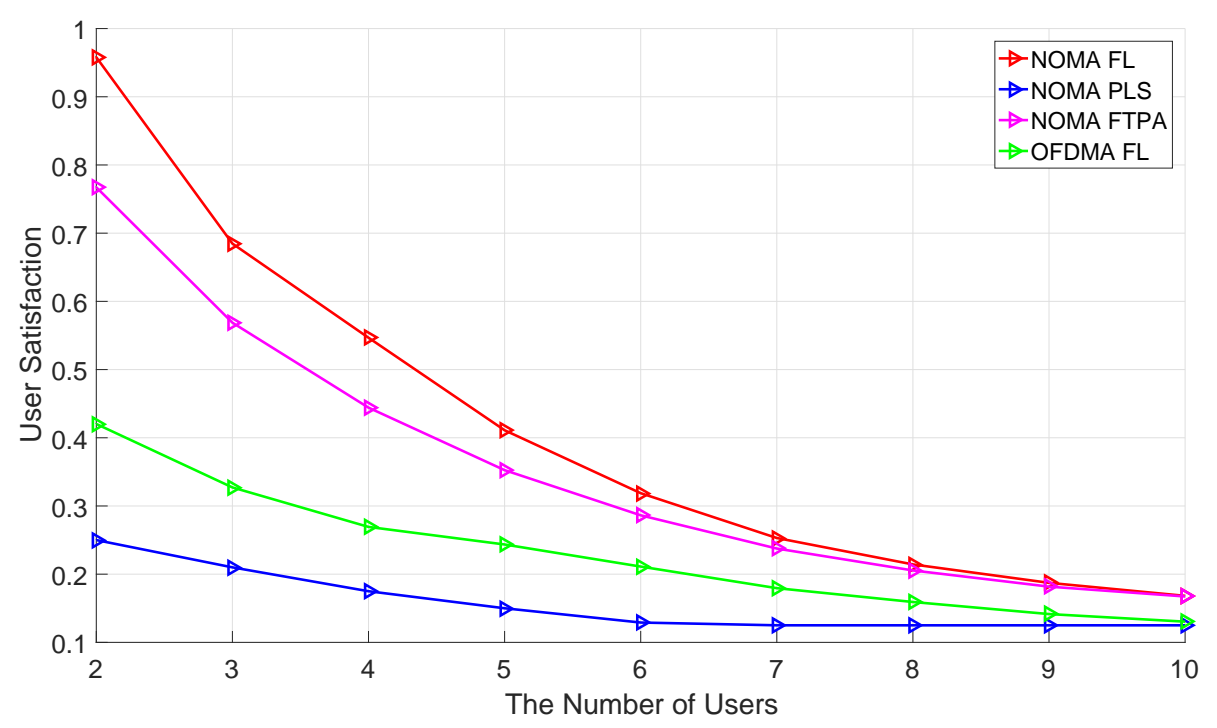

Figure 5. Comparisons of user satisfaction with the QoS expectation at $20 \mathrm{Mbps}$. 


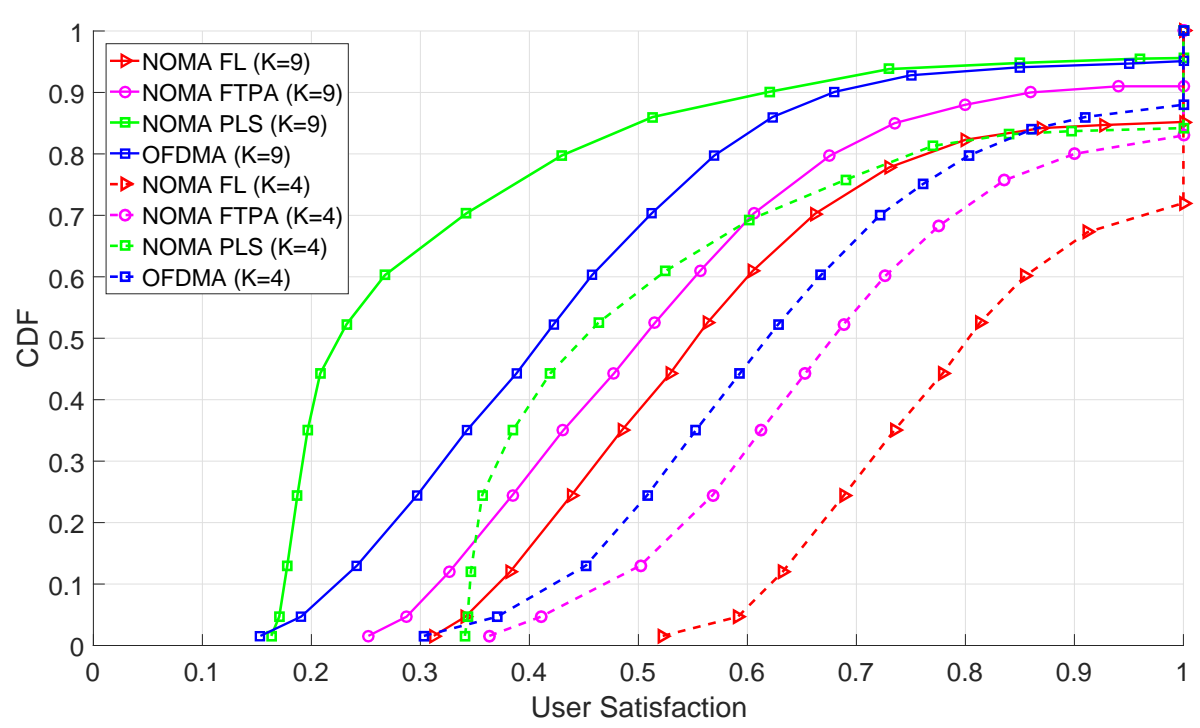

Figure 6. Comparisons of user satisfaction with the QoS expectation at $60 \mathrm{Mbps}$.

\subsubsection{QoS Variety}

When the QoS expectations are varied, Figure 7 shows that the performance gaps among comparisons will decrease by increasing the number of users. In UDN modeling, the room size, as shown in Table 3, is $36 \mathrm{~m}^{2}$. For high user density, when the number of users is greater than 9, NOMA FL can reach the AUDR of FTPA. This means that NOMA FL is applicable to the proposed VLC UDN scenario. As shown in Figure 8, with the normalization of capacity, our proposed method outperformed PLS and reached the performance level of FTPA under UDN conditions. In Figures 6-8, NOMA FL and OFDMA FL based on dynamic user priority are shown to be adaptive to changes in QoS requirements which are different from the fluctuations in PLS and FTPA based on static user priority.

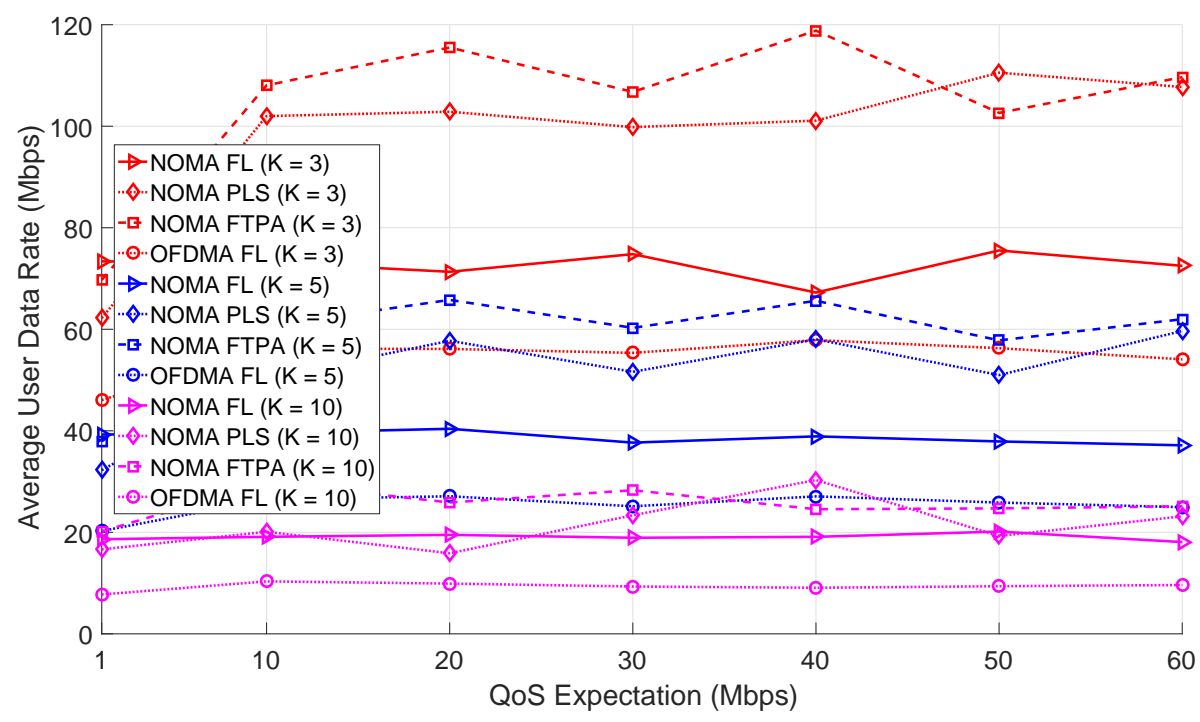

Figure 7. Comparisons of AUDR with varied QoS expectations and number of users. 


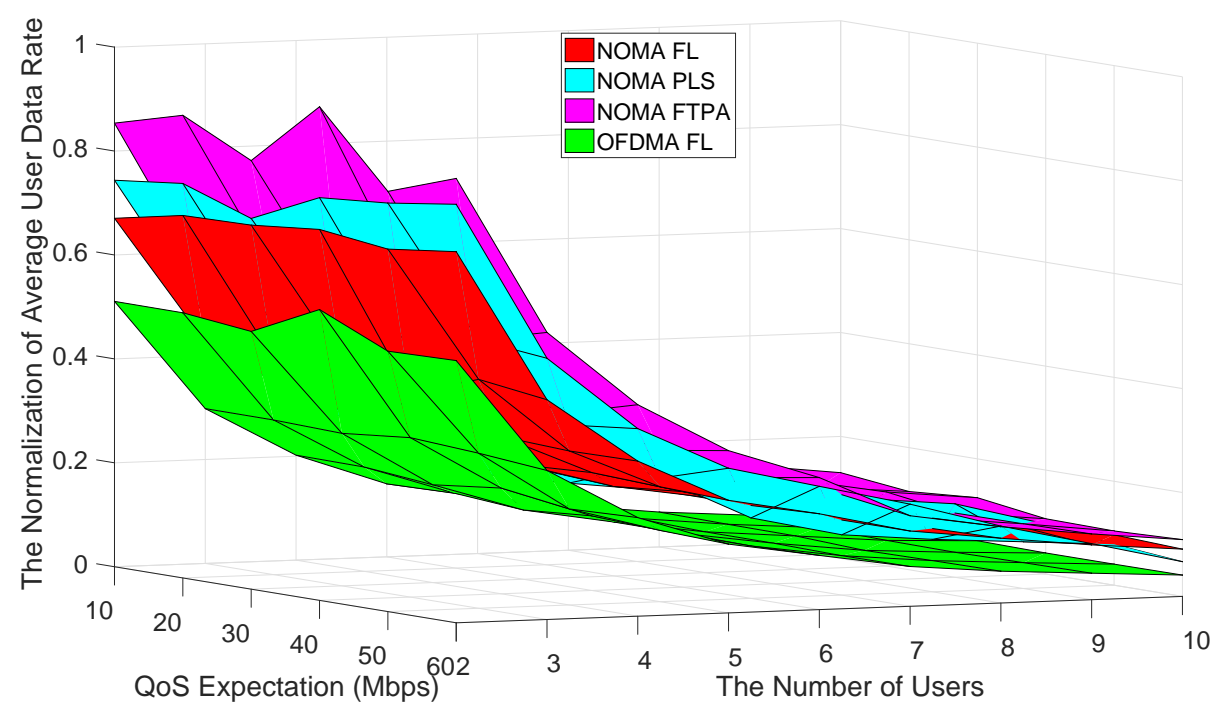

Figure 8. 3D plot with varied QoS, number of users, and AUDR.

\subsubsection{Fairness}

To further determine the tradeoff between the guarantee of a desired user data rate and system throughput, we developed the graph in Figure 9 to indicate that the Jain fairness based on PLS and FTPA is worse than in our proposed NOMA FL model. Furthermore, the max-min fairness based on NOMA FL is greater than PLS and OFDMA FL. Due to the power law ratio, PLS exacerbates imbalances among the power allocation of users, whereas FTPA alleviates inhomogeneous power allocation. In Figures 4 and 9, NOMA FL is shown to exchange a certain degree of enhancement of system throughput for the fairness of the QoS guarantee. It is clear that NOMA FL outperforms the other methods in terms of fairness without much decrease in system throughput. Particularly under UDN conditions, NOMA FL was shown to reach the performance level of PLS and FTPA if data rate maximization is regarded as the static target. For dynamic user priority, NOMA FL surpassed OFDMA FL in both system throughput and fairness.

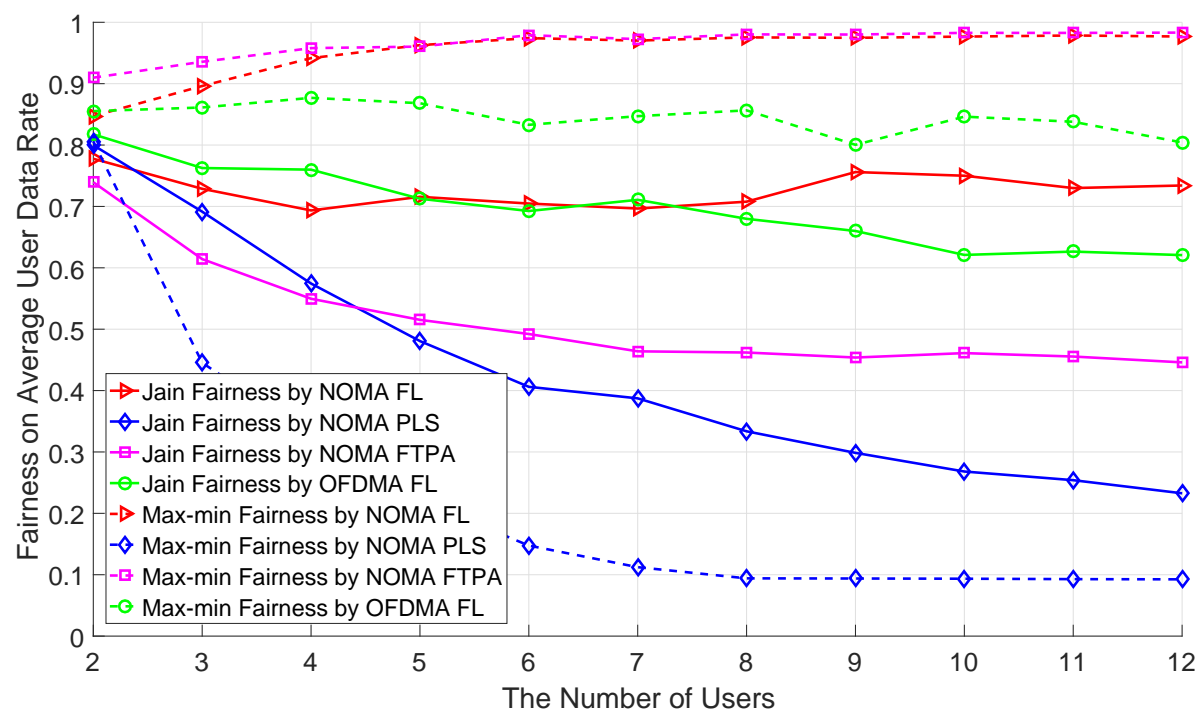

Figure 9. Comparison of fairness with a QoS expectation of $30 \mathrm{Mbps}$. 


\subsection{Case 3: Experimental Analysis of VLC Lighting}

When the experimental parameters in this case were listed in Table 3, except $\psi_{F O V}$ varied between $\psi_{F O V} \in\left\{45^{\circ}, 55^{\circ}, 65^{\circ}\right\}$, Figure 8 was yielded. This shows the effect of different illumination scenarios. When the FOV decreased, each method improved the AUDR because the increase of an optical concentrator gain caused the enhancement of the user data rate. For high user density, when $\psi_{F O V}=$ $45^{\circ}$ and the number of users exceeded 10, NOMA FL achieved the best AUDR. Furthermore, for a high optical AP density, the ISD of optical devices indoors may be less than 10 metres. Hence, a reduced FOV, such as $\psi_{F O V}=45^{\circ}$, may be applicative to realistic UDN scenario. This experimental result, shown in Figure 10, is similar to the conclusion generated in Figure 6, whereby a decrease of FOV under UDN conditions may lead to the best AUDR through our methods. Furthermore, we note that a decrease in FOV leads to the contractible attocell and restricts the coverage of VLC small cells. Hence, for AUDR alone, NOMA FL may lead to an increase that happens in special illumination cases.

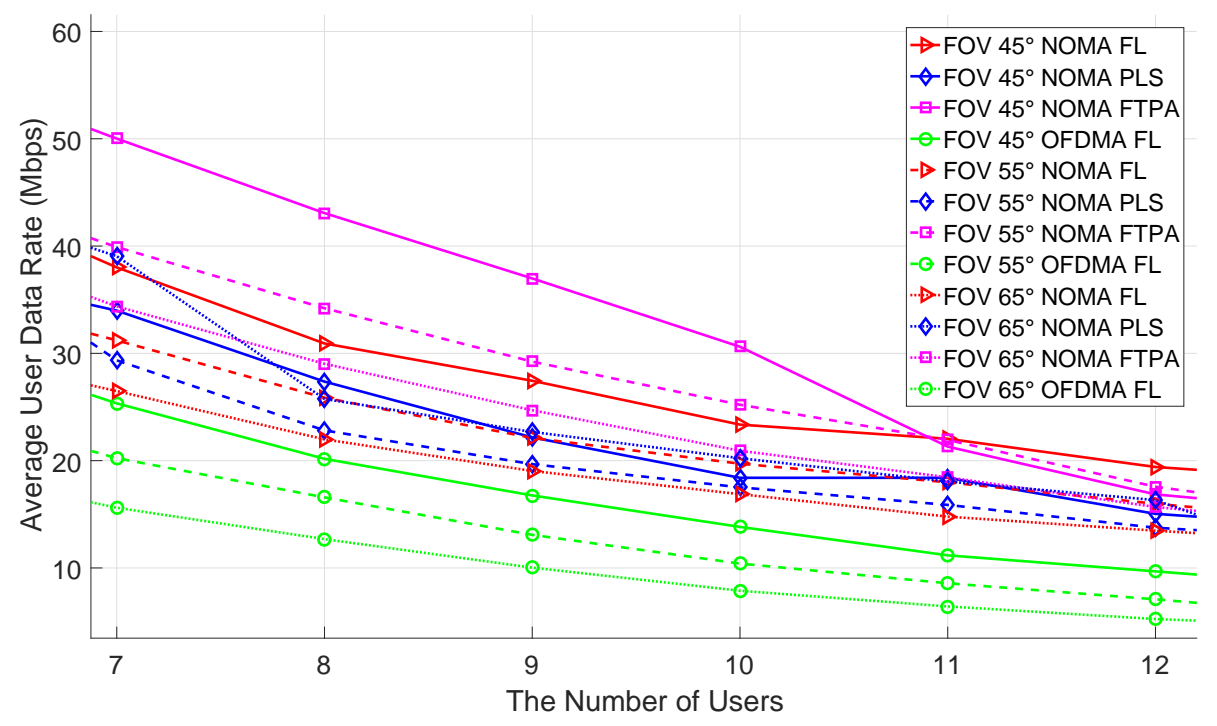

Figure 10. Comparisons of AUDR with varied field of views (FOV) and QoS expectations for $30 \mathrm{Mbps.}$

\section{Conclusions}

In this paper, we exploited multi-dimensional information to consider VLC user communication states. With NOMA, we introduced strong relevant features, including channel gains, the desired data rate, and outage probability. Using multiple features in FL systems, we comprehensively performed NOMA GRPA based on dynamic user priority and conducted a tradeoff between throughput and QoS fairness maximization. First, for the system throughput and fairness, the AUDR of our proposed method (NOMA FL) was shown to be greater than that of PLS in UDN conditions and OFDMA FL without limited UDN conditions. Second, although the AUDR of NOMA FL approached that of FTPA, our method showed better results in user satisfaction than those of three other studies. Finally, in a small-cell illumination scenario with UDN conditions, the AUDR of our method may achieve the best performance. In future work, the relationship between the semi-angle of the LED and NOMA-VLC FL should be investigated. A performance analysis of the interference components in FL could be also developed.

Author Contributions: All authors contributed to this paper. S.T. and Q.L. proposed the idea. S.T. and X.B. developed the framework of this paper. S.T. performed the experiments and analysed the simulation data. H.Y., Q.L., and Y.T. provided the laboratory and financial funding support. S.T. prepared the original draft of this paper. H.Y., Q.L., X.B. and Y.T. revised this paper.

Funding: This research was funded by National Natural Science Foundation of China (Grant No.61671477). 
Acknowledgments: This work was supported by the National Natural Science Foundation of China (No.61671477). The authors wish to acknowledge the valuable technical support of M.Phil. Xiangwei Bai.

Conflicts of Interest: The authors declare no conflict of interest.

\section{Abbreviations}

The following abbreviations are used in this manuscript:

$\begin{array}{ll}\text { AP } & \text { Access point } \\ \text { AUDR } & \text { Average user data rate } \\ \text { CDF } & \text { Cumulative distribution function } \\ \text { FL } & \text { Fuzzy logic } \\ \text { FOV } & \text { Field of vision } \\ \text { FTPA } & \text { Fractional transmit power allocation } \\ \text { GRPA } & \text { Gain ratio power allocation } \\ \text { ISD } & \text { Inter-site distance } \\ \text { ITU-R } & \text { International telecommunications union-radio } \\ \text { LED } & \text { Light-emitting diode } \\ \text { LiFi } & \text { Light fidelity } \\ \text { LOS } & \text { Line of sight } \\ \text { Mbps } & \text { Megabits per second } \\ \text { MF } & \text { Membership function } \\ \text { NOMA } & \text { Non-orthogonal multiple access } \\ \text { OFDMA } & \text { Orthogonal frequency division multiple access } \\ \text { OMA } & \text { Orthogonal multiple access } \\ \text { OOK } & \text { On-off keying } \\ \text { OWC } & \text { Optical wireless communication } \\ \text { PDF } & \text { Probability density function } \\ \text { PLS } & \text { Power law strategy } \\ \text { QoS } & \text { Quality of service } \\ \text { SIC } & \text { Successive interference cancellation } \\ \text { SINR } & \text { Signal interference plus noise ratio } \\ \text { UDN } & \text { Ultra dense network } \\ \text { VLC } & \text { Visible light communication } \\ \text { WiFi } & \text { Wireless fidelity } \\ & \end{array}$

\section{References}

1. Chen, S.; Qin, F.; Hu, B.; Li, X.; Chen, Z.; Liu, J. User-Centric Ultra-Dense Networks for 5G; Springer: Cham, Switzerland, 2018; pp. 1-3.

2. Chen, S.; Qin, F.; Hu, B.; Li, X.; Chen, Z. User-centric ultra-dense networks for 5G: Challenges, methodologies, and directions. IEEE Wirel. Commun. 2016, 23, 78-85. [CrossRef]

3. Haas, H.; Yin, L.; Wang, Y.; Chen, C. What is lifi? J. Lightw. Technol. 2016, 34, 1533-1544. [CrossRef]

4. Yin, L.; Popoola, W.O.; Wu, X.; Haas, H. Performance evaluation of non-orthogonal multiple access in visible light communication. IEEE Trans. Commun. 2016, 64, 5162-5175. [CrossRef]

5. Ding, Z.; Dai, H.; Poor, H.V. Relay selection for cooperative NOMA. IEEE Wirel. Commun. Lett. 2016, 5, 416-419. [CrossRef]

6. Zhang, X.; Gao, Q.; Gong, C.; Xu, Z. User grouping and power allocation for NOMA visible light communication multi-cell networks. IEEE Commun. Lett. 2017, 21, 777-780. [CrossRef]

7. Marshoud, H.; Kapinas, V.M.; Karagiannidis, G.K.; Muhaidat, S. Non-orthogonal multiple access for visible light communications. IEEE Photonics Technol. Lett. 2016, 28, 51-54. [CrossRef]

8. Mitra, R.; Bhatia, V. Precoded Chebyshev-NLMS-Based Pre-Distorter for Nonlinear LED Compensation in NOMA-VLC. IEEE Trans. Commun. 2017, 65, 4845-4856. [CrossRef]

9. Chen, C.; Zhong, W.D.; Yang, H.; Du, P. On the performance of MIMO-NOMA-based visible light communication systems. IEEE Photonics Technol. Lett. 2018, 30, 307-310. [CrossRef] 
10. Jia, K.; Hao, L.; Bai, L.; Yu, C. Indoor Visible Light Communication System Based on Non-Orthogonal Multiple Access. Acta Opt. Sin. 2017, 37, 70-80. [CrossRef]

11. El-Sayed, M.M.; Ibrahim, A.S.; Khairy, M.M. Power allocation strategies for Non-Orthogonal Multiple Access. In Proceedings of the 2016 International Conference on Selected Topics in Mobile and Wireless (MoWNeT), Cairo, Egypt, 11-13 Apirl 2016; pp. 1-6. [CrossRef]

12. Hojeij, M.R.; Farah, J.; Nour, C.A.; Douillard, C. Resource allocation in downlink non-orthogonal multiple access (NOMA) for future radio access. In Proceedings of the 2015 IEEE 81st Vehicular Technology Conference (VTC Spring), Glasgow, UK, 11-14 May 2015; pp. 1-6. [CrossRef]

13. Saito, Y.; Benjebbour, A.; Kishiyama, Y.; Nakamura, T. System-level performance evaluation of downlink non-orthogonal multiple access (NOMA). In Proceedings of the 2013 IEEE 24th International Symposium on Personal Indoor and Mobile Radio Communications (PIMRC), London, UK, 8-11 September 2013; pp. 611-615. [CrossRef]

14. Benjebbour, A.; Li, A.; Saito, Y.; Kishiyama, Y.; Harada, A.; Nakamura, T. System-level performance of downlink NOMA for future LTE enhancements. In Proceedings of the 2013 IEEE Globecom Workshops (GC Wkshps), Atlanta, GA, USA, 9-13 December 2013; pp. 66-70. [CrossRef]

15. Hojeij, M.R.; Farah, J.; Nour, C.A.; Douillard, C. New optimal and suboptimal resource allocation techniques for downlink non-orthogonal multiple access. Wirel. Pers. Commun. 2016, 87, 837-867. [CrossRef]

16. Oviedo, J.A.; Sadjadpour, H.R. A new NOMA approach for fair power allocation. In Proceedings of the 2016 IEEE Conference on Computer Communications Workshops (INFOCOM WKSHPS), San Francisco, CA, USA, 10-14 April 2016; pp. 843-847. [CrossRef]

17. Cao, Y.; Yang, Z.; Feng, Y. New NOMA power allocation strategy. J. Commun. 2017, 38, 157-165. [CrossRef]

18. Yapici, Y.; Guvenc, I. Non-Orthogonal Multiple Access for Mobile VLC Networks with Random Receiver Orientation. arXiv 2018, arXiv:1801.04888.

19. Sediq, A.B.; Gohary, R.H.; Schoenen, R.; Yanikomeroglu, H. Optimal tradeoff between sum-rate efficiency and Jain's fairness index in resource allocation. IEEE Trans. Wirel. Commun. 2013, 12, 3496-3509. [CrossRef]

20. Li, X.; Zhang, R.; Wang, J.; Hanzo, L. Cell-Centric and User-Centric Multi-User Scheduling in Visible Light Communication aided networks. In Proceedings of the IEEE International Conference on Communications (ICC), London, UK, 8-12 June 2015; pp. 5120-5125. [CrossRef]

21. Wang, Y.; Wu, X.; Haas, H. Fuzzy logic based dynamic handover scheme for indoor Li-Fi and RF hybrid network. In Proceedings of the 2016 IEEE International Conference on Communications (ICC), Kuala Lumpur, Malaysia, 22-27 May 2016; pp. 1-6. [CrossRef]

22. Jin, F.; Zhang, R.; Hanzo, L. Resource Allocation Under Delay-Guarantee Constraints for Heterogeneous Visible-Light and RF Femtocell. IEEE Trans. Wirel. Commun. 2015, 14, 1020-1034. [CrossRef]

23. Jin, F.; Li, X.; Zhang, R.; Dong, C.; Hanzo, L. Resource allocation under delay-guarantee constraints for visible-light communication. IEEE Access 2016, 4, 7301-7312. [CrossRef]

24. Cui, J.; Ding, Z.; Fan, P. A novel power allocation scheme under outage constraints in NOMA systems. IEEE Signal Process. Lett. 2016, 23, 1226-1230. [CrossRef]

25. Hou, J.; O'Brien, D.C. Vertical handover-decision-making algorithm using fuzzy logic for the integrated Radio-and-OW system. IEEE Trans. Wirel. Commun. 2006, 5, 176-185. [CrossRef]

26. Bu, R.; Wang, S.; Wang, H. Fuzzy logic vector-based forwarding routing protocol for underwater acoustic sensor networks. Trans. Emerg. Telecommun. Technol. 2018, 29, e352. [CrossRef]

27. Jain, R.K.; Chiu, D.M.W.; Hawe, W.R. A Quantitative Measure of Fairness and Discrimination; Eastern Research Laboratory, Digital Equipment Corporation: Hudson, MA, USA, 1984; pp. 2-7.

28. Wu, X.; Safari, M.; Haas, H. Three-state fuzzy logic method on resource allocation for small cell networks. In Proceedings of the 2015 IEEE 26th Annual International Symposium on Personal, Indoor, and Mobile Radio Communications (PIMRC), Hong Kong, China, 30 August-2 September 2015; pp. 1168-1172. [CrossRef]

(C) 2018 by the authors. Licensee MDPI, Basel, Switzerland. This article is an open access article distributed under the terms and conditions of the Creative Commons Attribution (CC BY) license (http:/ / creativecommons.org/licenses/by/4.0/). 